\title{
CHINA Y MÉXICO 2012-2018, ENTRE EL INICIO IDÍLICO Y LA REALIDAD
}

\author{
CHINA AND MEXICO 2012-2018, BETWEEN \\ THE IDYLLIC START AND THE REALITY
}

LA CHINE ET LE MEXIQUE: DE LA NAISSANCE D’UNE
IDYLLE À LA DÉCOUVERTE DE LA RÉALITÉ (2012-2018)

ROMER CORNEJO

El Colegio de México

rcornejo@colmex.mx

Resumen: Las relaciones de México con China en el sexenio 2012-2018 no tuvieron cambios importantes en comparación con los gobiernos anteriores. México continuó con la política de no considerar el peso de China en la comunidad internacional, ni las características de su sistema político ni sus proyectos económicos de gran potencia. Si bien las relaciones entre ambos países dieron inicio con la consigna de "relanzamiento", se vieron lesionadas por los fracasos de los proyectos económicos chinos en México. Las relaciones diplomáticas siguieron su curso normal, con encuentros entre funcionarios de diversos niveles y firma de interesantes acuerdos de cooperación. Por su parte, China mantuvo una eficaz política cultural de creación de opinión pública favorable entre académicos, políticos y medios de comunicación

Palabras clave. relación México-China; corrupción; tren MéxicoQuerétaro; Dragon Mart; política exterior; cooperación.

Abstract: Mexico's relations with China during the 2012-2018 administration saw no significant change compared to previous governments. Mexico continued its approach of failing to take into account China's weight in the international community, the characteristics of its political system, or its powerful economic projects. Although the relations between both countries began with the slogan of "resetting," they were hurt by the failures of Chinese economic projects in Mexico. Diplomatic relations followed their usual course, with meetings between officials at various levels and the signing of interesting cooperation agreements. For its part, China maintained an effective 
cultural policy to create favorable public opinion among academics, politicians and the media.

Keywords: Mexico-China relationship; corruption; Mexico-Querétaro train; Dragon Mart; foreign policy; cooperation.

\section{Traducción de Gonzalo Celorio Morayta}

RÉSumÉ: Entre 2012 et 2018, les relations Mexique-Chine n'ont pas beaucoup changé par rapport aux périodes présidentielles mexicaines précédentes. Le Mexique s'est encore obstiné à ne pas reconnaitre l'importance de la Chine dans la communauté internationale, ni les particularités de son système politique, ni ses projets économiques de grande puissance. Même si le gouvernement de Peña Nieto a donné la consigne de «relancer» les rapports avec la Chine, ce dessein a souffert de l'échec de certains projets économiques chinois au Mexique. Les rapports diplomatiques ont suivi leur cours normalement, grâce aux rencontres de fonctionnaires de plusieurs niveaux et à la conclusion d'accords assez intéressants pour faciliter la coopération. Quant à la Chine, elle a poursuivi sa politique culturelle très efficace qui cherche à cultiver l'opinion favorable des milieux universitaires et politiques, ainsi que celle des médias.

Mots clefs: Rapports Mexique-Chine; corruption; train MexicoQuerétaro; Dragon Mart; politique étrangère; coopération.

Traducción de Bernardo Mabire

Fecha de recepción: diciembre de 2018

Fecha de aceptación: febrero de 2019 
$\mathrm{E}$ N el sexenio 2012-2018, las relaciones entre China y México arrancaron con enorme ímpetu, coincidiendo con el ascenso al poder de los presidentes de ambos países que, en sus primeros encuentros, mostraron un gran acercamiento personal; explotaron su entusiasmo de reformas y hasta el hecho de que sus cónyuges provenían del medio artístico y eran ampliamente conocidas en sus respectivos países. Debido a los fracasos de dos grandes proyectos económicos de China, atribuibles a malos manejos en México, el entusiasmo inicial se apagó pronto y las relaciones quedaron en un nivel muy bajo.

Ambos gobernantes, Enrique Peña Nieto y Xi Jinping, empezaron sus gobiernos con proyectos de transformación. El primero, prometiendo renovación en su partido político y buscando el consenso para sus políticas a través del llamado Pacto por México, que incluía una amplia iniciativa de reformas estructurales, además de plantear la construcción de grandes obras de infraestructura que detonarían el estancado crecimiento del país. El segundo dio inicio con una respuesta para la situación política y geoeconómica de México: en el ámbito interno, una gran campaña contra la corrupción a la par de medidas de estímulo dirigidas al mercado interno y, hacia el exterior, un ambicioso plan de posicionamiento global, el "Cinturón y la Ruta", que abarca desde el ámbito cultural hasta grandes inversiones en infraestructura que colocarían al país como centro global de comunicaciones y comercio.

En México, las expectativas de reformas pronto se empantanaron debido a grandes escándalos de corrupción, unos precisamente vinculados a la relación económica con China y otros a la desaparición irresuelta de 43 estudiantes normalistas en Ayotzinapa, una empobrecida zona del estado de Guerrero, junto al aumento desmedido de la violencia y la corrupción en el país. Todo esto dejó al gobierno sumido en el desprestigio, pero las alianzas previamente pactadas lograron la aprobación de la mayoría de las reformas prometidas en el Congreso, como la energética y la de educación. El des- 
prestigio fue tal, que el partido de gobierno perdió las elecciones de 2018 por un enorme margen. En el caso de China, la lucha contra la corrupción ha tenido una eficacia relativa, por lo menos debido al miedo político que implica ser descubierto, aunque en varios casos se usó para deshacerse de enemigos políticos (que no estaban exentos de actos de corrupción). Éste fue el marco necesario para el afianzamiento y la concentración del poder en la figura de Xi Jinping, quien logró en marzo de 2018 reformar la constitución para poder reelegirse más de una vez, lo cual se suma a la consolidación de un sistema de culto a la personalidad que recuerda al de Mao Zedong, con las diferencias del caso. Por su parte, el proyecto del "Cinturón y la Ruta" ha continuado avanzando, no sin algunos contratiempos, conectando a China por tren con el centro, sur y sudeste de Asia, con Europa y más recientemente ha proyectado una conexión marítima con África, América Latina y el Ártico. Ciertamente no es un proyecto terminado, pero ha logrado invertir o colocar como deuda gran parte de sus enormes reservas y expandir la presencia de sus empresas en el mundo. El proyecto se ha acompañado de una enorme campaña de propaganda entre académicos y personas que influyen en la opinión pública, muy evidente en México y el resto de América Latina.

En este contexto, una vez más, la relación de México con China puede caracterizarse porque se integra por un país que carece de una clara definición de su política exterior en función de sus grandes problemas internos y su proyección internacional, con otro que parte de la solución de sus asuntos internos, perfectamente delimitados, y con planes precisos a mediano y largo plazo respecto a su posicionamiento como potencia global. La administración del gobierno mexicano, al igual que en el pasado, no quiso reconocer que China es la segunda potencia mundial y apenas la esgrimió como “culpable" de competencia económica y responsable del alza de los precios de alimentos. 
En el Plan Nacional de Desarrollo (PND) 2013-2018 del gobierno mexicano, ${ }^{1}$ China primero se menciona como causante del aumento de los precios de los alimentos debido al incremento de su demanda, lo cual incidió, según el Plan, en el aumento del precio de la canasta básica en México. Asimismo, se hace referencia a la competencia de la mano de obra de China desde el ingreso de ese país a la Organización Mundial de Comercio (OMC), como una de las causas de la baja de competitividad de la mano de obra mexicana, aunque reconoce que el reciente aumento de los salarios en el país asiático podría beneficiar a México. ${ }^{2}$ En el PND, México aparece comparado en forma desfavorable frente China en el desempeño en la prueba PISA (Programa Internacional de Evaluación de Alumnos), así como en la inversión en investigación y desarrollo, pública y privada. ${ }^{3}$ Igualmente, reconoce la necesidad de atraer turismo de China y afirma, de la manera más vaga posible, que "México tiene el reto de llevar las relaciones con dicho país hacia un nuevo paradigma de cooperación y diálogo, que permita propiciar nuevos esquemas de entendimiento e intercambio". ${ }^{4}$ Una afirmación que puede hacerse sobre cada país del mundo.

En el presente artículo presentaré primero el estado y desarrollo de las relaciones económicas y luego las relaciones diplomáticas, donde incluiré una breve referencia a las relaciones culturales entre ambos países.

${ }^{1}$ Véase texto completo, Gobierno de la República, Plan Nacional de Desarrollo 2013-2018. Diario Oficial de la Federación, 20 de mayo de 2013, http:/ / www.dof.gob.mx/nota_detalle.php?codigo=5299465\&fecha= 20/05/2013

${ }^{2}$ Loc. cit.

${ }^{3}$ Loc. cit.

${ }^{4}$ Loc. cit. 
LAS RELACIONES ECONÓMICAS, EL DÉFICIT, LA COMPETENCIA Y LOS FRACASOS DE LOS PROYECTOS DE INVERSIÓN

El intercambio económico entre México y China mantuvo una tendencia al alza y paralelamente un crecimiento consecuente del déficit para México. La composición de las importaciones de México tuvo un ligero cambio respecto al aumento de bienes intermedios. La gran preocupación en varios sectores del país fue el déficit comercial y el bajo valor agregado de las exportaciones mexicanas a China. Lo que se ha observado es un aumento de las exportaciones de carne de cerdo y de tequila. Pero el $74.5 \%$ de las importaciones originarias de China son de bienes intermedios, y $11.2 \%$ son bienes de capital, gran parte de los cuales se utilizan en las industrias automotriz y electrónica para exportarlos, ya ensamblados, en autos y dispositivos electrónicos, al mercado de Estados Unidos, y una pequeña parte se queda en el mercado mexicano, según Fernando Ruiz, director del Consejo Empresarial Mexicano de Comercio Exterior (COMcE). ${ }^{5}$ De acuerdo con la Secretaría de Agricultura (Sagarpa), las exportaciones del sector agropecuario al país asiático sumaron 321 mdd en 2017, lo que significó un incremento de $54 \%$. De acuerdo con Sagarpa, los 10 productos agroalimentarios que más exporta México a China son: cerveza, harina de pescado, aguacate, algodón, moluscos vivos, langostas, materias pécticas, cueros y pieles de bovino, camarones y langostinos congelados, y tequila. Como es obvio, estos productos difícilmente pueden modificar el déficit comercial.

Por su parte, tanto en el acero como en textiles y zapatos, hubo controversias por los bajos precios de los productos de China, a las que se sumaron protestas de los empresarios en Monclova y Guanajuato.

${ }^{5}$ Dainzú Patiño, “¿Cómo reducir el déficit comercial de México con China?", Expansión, 3 de agosto (s/a), https://expansion.mx/economia/ 2018/08/03/como-reducir-el-deficit-comercial-de-mexico-con-china (consulta del 12 de septiembre de 2018). 
La composición del comercio entre ambos países está dictada por las características de sus economías; ambos buscan colocar productos procesados en el mercado exterior, y China tiene una obvia ventaja comparativa con respecto a México en este sentido, dado su enorme desarrollo industrial y su inversión en ciencia, tecnología y desarrollo. El país asiático es un gran consumidor de bienes básicos tanto agrícolas como mineros y, además, tiene un claro proyecto de incorporación de un número importante de población pobre a su economía, de manera que la competencia en cuanto a la mano de obra es todavía imbatible para México. En este sentido, llama la atención la insistencia de gran parte de los textos escritos en México sobre la relación comercial entre ambos países, que propugnan por un estrechamiento de los vínculos comerciales sin considerar que éstos están regidos por el mercado, y que no se puede obligar a los empresarios a establecer relaciones que no consideren productivas, ni a un gobierno como el mexicano que ha renunciado cada vez más a su papel como ente económico a intervenir en ello. En todo caso, es digno reconocer que la Secretaría de Economía y, particularmente la de Relaciones Exteriores, han hecho un gran trabajo en establecer un marco institucional que facilite las relaciones, aun si éstas no se han expandido.

\section{CUADRo 1}

Balanza comercial de México con China (valores en miles de dólares)

\begin{tabular}{ccccc}
\hline Año & Exportaciones & Importaciones & Comercio total & Balance \\
\hline 2011 & 5964225 & 52248003 & 58212228 & -46283778 \\
2012 & 5720732 & 56936129 & 62656861 & -51215397 \\
2013 & 6468508 & 61321376 & 67789884 & -54852868 \\
2014 & 5964125 & 66255959 & 72220084 & -60291834 \\
2015 & 4873144 & 69987814 & 74860958 & -65114670 \\
2016 & 5411240 & 69520664 & 74931904 & -64109424 \\
2017 & 6712525 & 74145286 & 80857811 & -67432761 \\
\hline
\end{tabular}

Fuente: Subsecretaría de Comercio Exterior. 
CuAdro 2

Inversión directa de China en México (millones de dólares)

\begin{tabular}{llllll}
\hline 2011 & 2012 & 2013 & 2014 & 2015 & 2016 \\
\hline 38.1 & 102.5 & 54.9 & 100.7 & 52.9 & 56.9 \\
\hline
\end{tabular}

Fuente: https://www.gob.mx/cms/uploads/attachment/file/294 151/II-ComercioExterior-enero2018.pdf (consulta del 3 de junio de 2018).

Como puede observarse, la inversión de China en el país fue muy poco significativa, comparada con la inversión total en América Latina, que rebasa por mucho los 200000 millones de dólares. Asimismo, México estuvo atento a las acciones de dumping de China e impuso cuotas compensatorias a varios productos, en 2015 , ya concluido el asunto del tren:

\section{Cuadro 3}

Cuotas compensatorias definitivas impuestas por México

\begin{tabular}{ll}
\hline Producto & País \\
\hline Bicicletas & China \\
Fregaderos de acero inoxidable & China \\
Jaladeras de acero y de zamac & China \\
Lámina rolada en frío & China \\
Rollos de acero laminados en caliente & Alemania, China y Francia \\
Sulfato de amonio & China y EEuU \\
\hline
\end{tabular}

Fuente: Secretaría de Economía. 


\section{Cuadro 4 \\ Investigaciones antidumping iniciadas por México}

\begin{tabular}{ll}
\hline Producto & País \\
\hline Aceros planos recubiertos & China y Taiwán \\
Alambrón de acero & China \\
Artículos para cocinar de aluminio & China \\
Ferrosilicomanganeso & India \\
Productos de presfuerzo & China, España y Portugal \\
Recubrimientos cerámicos para muros y pisos & China \\
\hline
\end{tabular}

Fuente: Secretaría de Economía.

\section{CuAdro 5 \\ Cuotas compensatorias definitivas}

\begin{tabular}{ll}
\hline Producto & País \\
\hline Bicicletas & China \\
Fregaderos de acero inoxidable & China \\
Jaladeras de acero y de zamac & China \\
Lámina rolada en frío & China \\
Rollos de acero laminados en caliente & Alemania, China y Francia \\
Sulfato de amonio & China y EEuU \\
\hline
\end{tabular}

Fuente: Secretaría de Economía.

\section{El caso del Dragon Mart}

El Dragon Mart de Cancún fue un proyecto de empresas chinas para el establecimiento de un enorme centro de distribución de mercancías en la costa del sur de México, cuyo tamaño superaría a uno previamente establecido por el gobierno del país asiático en Dubái. Según una nota aparecida en el diario oficial China Daily, en 2012, ${ }^{6}$ este proyecto tenía

${ }^{6} \mathrm{Hu}$ Yuanyuan, "Plans afoot to open second «Dragon Mart» commercial center in Mexico", China Daily, 23 de febrero de 2012, http://global. chinadaily.com.cn/a/201202/23/WS5a2f5468a3108bc8c6723337.html (consulta del 14 de julio de 2018). 
el apoyo de la Oficina de Desarrollo del Comercio Internacional perteneciente al Ministerio de Comercio de aquel país, y sería desarrollado por las compañías Chinamex Middle East Investment \& Trade Promotion Center Co Ltd y Chengkai (Beijing) Investment Co Ltd.

El apoyo de China al establecimiento de este centro continuó al año siguiente. Según el China Daily, “...el Dragon Mart de Cancún contribuiría no sólo a ayudar a los inversionistas chinos sino, también a los comerciantes internacionales a explorar el mercado latinoamericano". ${ }^{7}$ Yu Yanqing, representante de Chengkai Beijing (Investment) Co Ltd, declaró al citado periódico: "Estamos planeando ajustar las estrategias del Dragon Mart en Cancún para diversificar los sectores de nuestros negocios". ${ }^{8}$ La nota del China Daily agregó:

Apoyados por el Ministerio de Comercio (de China) y el gobierno mexicano, la construcción del Dragon Mart empezó en 2012, y ambos gobiernos han acordado establecer el proyecto como la plataforma de comercio de bienes chinos más grande de América Latina [...] Con una inversión total de más de 10 mil millones de yuanes (1.63 mil millones de dólares) el proyecto es un desarrollo conjunto de tres compañías: Chinamex Middle East Investment \& Trade Promotion Center Co Ltd, Chengkai (Beijing) Investment Co Ltd and New Rainbow (China) Limited. ${ }^{9}$

La misma fuente reconoce: "Sin embargo, debido a la oposición del público, la construcción del proyecto ha sido pospuesta desde el fin del año pasado". ${ }^{10}$ Yu Yanqing afirmó que la compañía Chengkai había invertido al menos 300 millones de yuanes en el proyecto y más del $30 \%$ había sido comple-

${ }^{7}$ Bao Chang, "Dragon Mart to land in Cancun", China Daily, 7 de junio de 2013, http://www.chinadaily.com.cn/china/2013xivisit/2013-06/ 07/content_16580627.htm (consulta del 3 de julio de 2013).

\footnotetext{
${ }^{8}$ Loc. cit.

${ }^{9}$ Loc. cit.

${ }^{10}$ Loc. cit.
} 
tado. Asimismo, declaró que debido a las objeciones locales en Cancún, se había decidido desarrollar el proyecto como un centro de comercio internacional más que como un centro de mercancías de China. El China Daily agregó que se cree que la oposición al proyecto proviene de comerciantes extranjeros de Estados Unidos, Corea del Sur e India, dominantes en el comercio de la región, que tienen la expectativa de que las mercancías chinas sean más competitivas. Por ello, Yu Yanqing afirmó: "Los productos de comerciantes internacionales de Estados Unidos, Japón, y Corea del Sur serían no menos del $30 \%$ de los bienes vendidos en el centro comercial". ${ }^{11}$

Mientras tanto, el presidente de China, Xi Jinping, declaraba en su entusiasta visita a principios de junio al país que él y el presidente de México habían decidido llevar las relaciones bilaterales al rango de "asociación estratégica integral" en beneficio de un crecimiento continuo de las relaciones bilaterales con un alcance más amplio sobre una plataforma mayor, para así elevar las relaciones a un nuevo estadio de desarrollo. ${ }^{12}$

En México, las autoridades locales de Cancún, lideradas por el Partido de la Revolución Democrática (PRD), junto a una opinión pública más amplia, alegaron que el citado proyecto provocaría serios daños ambientales, pues contemplaba 722 viviendas, veinte naves comerciales y un total de 3000 locales, en una superficie de 561 hectáreas. Además, según algunos grupos empresariales, disminuirían los precios de las manufacturas de China entre 10 y 15 por ciento. A otros grupos empresariales esto les pareció una competencia desleal. Dada la exaltación de la opinión pública, en la que algunos sectores revivieron el racismo tradicional antichino del país y colocaban como uno de los males del proyecto el hecho de

11 Loc. cit.

12 "China-Mexico ties face unprecedented opportunity: Xi”, China Daily, 6 de junio de 2013, http:/ /www.chinadaily.com.cn/china/2013xivi sit/2013-06/06/content_16577448.htm (consulta del 3 de julio de 2013). 
que más de mil familias chinas vendrían a vivir a México, una publicación digital resumió la opinión de muchos:

Y si fuera poco el daño causado al ecosistema, se espera en principio, la llegada de más de 2 mil 500 comerciantes chinos que vivirán dentro del complejo Dragon Mart; la experiencia indica que con el paso de los años traerán a familiares, esposas e hijos, lo que desembocará en un núcleo poblacional de aproximadamente 100 mil chinos, que conformarán el primer barrio chino del Caribe mexicano, similar al barrio Chino del D.F en México, o al barrio Chino de Los Ángeles, California, cuyas principales características son la endogamia y resistencia a integrarse a la cultura anfitriona, causando una mancha cultural que podría afectar las tradiciones y creencias nacionales de la zona. ${ }^{13}$

El propio lado chino fue dejando el proyecto en manos de un grupo mexicano hasta que el gobierno federal decretó su anulación en enero de 2015, debido a la presión de un sector empresarial, de ecologistas y a la intensa militancia del PRD. El 8 de enero, un tribunal colegiado declaró infundado el amparo promovido por la empresa y el 21 de enero, el juzgado de distrito emitió el acuerdo correspondiente para dar por concluido el juicio de amparo. ${ }^{14}$

13 "Dragon Mart Cancún: la destrucción del monstruo chino llegó a México", 21 de noviembre de 2012, https://ecoosfera.com/2012/11/dra gon-mart-cancun-la-destruccion-del-monstruo-chino-llego-a-mexico/ (consulta del 3 de diciembre de 2012). Véase también Ernestina McDonald, "Dragón Mart, la invasión china a Cancún", Noticieros Televisa, 6 de diciembre de 2012, http://noticierostelevisa.esmas.com/especiales/5340 81/dragon-mart-invasion-china-cancun/ (consulta del 15 de diciembre de 2012).

${ }^{14}$ Víctor Chávez, "Cancelación de Dragon Mart, en respuesta a demanda ciudadana: PRD”, El Financiero, 26 de enero de 2015, http://www. elfinanciero.com.mx/economia/cancelacion-de-dragon-mart-en-respues ta-a-demanda-ciudadana-prd (consulta del 27 de enero de 2015). Véase también Ivan Restrepo, "Dragon Mart, negocio oscuro", La Jornada, 2 de 
Si bien algunas voces de la Universidad Nacional Autónoma de México (UnAM), como el especialista Enrique Dussel Peters, consideró un error el finiquito del proyecto por no haber calculado las consecuencias ambientales en su momento, el sector industrial agrupado en la Confederación de Cámaras Industriales (Concamin) apoyó su cancelación. Ysmael López, presidente de la Comisión de Dragon Mart de la Concamin, dijo que la clausura del proyecto era una decisión positiva, no sólo por el bien de la industria, sino del país en general. Añadió que si el proyecto se hubiera concretado "habrían entrado 300 mil toneladas de mercancías chinas a México al mes, la mayoría a precios inferiores al valor real, poniendo en riesgo miles de empleos". ${ }^{15}$

El proyecto Dragon Mart, como lo presentó la prensa oficial de China (lo que no es poca cosa), con gran apoyo de sectores del gobierno y empresas de ese país, como se reseñó líneas arriba, en sus últimos tiempos y de una manera muy poco clara, pasó a ser presentado como propiedad de empresas mexicanas, con $10 \%$ de inversión de China. ${ }^{16}$ En esa tónica, en junio de 2013, el embajador de China en México, Zeng Gang, afirmó sobre el fallido proyecto del Dragon Mart que el gobierno de China no tenía conocimiento porque se trataba de un proyecto de empresarios mexicanos, con apenas un

febrero de 2015, https://www.jornada.com.mx/2015/02/02/opinion/01 6a2pol (consulta del 3 de febrero de 2015).

15 Marcela Ojeda e Isabel Becerril, "Ven daño a relación México-China por Dragon Mart", El Financiero, 27 de enero de 2015, http:/ /www.elfi nanciero.com.mx/economia/ven-dano-a-relacion-mexico-china-por-dra gon-mart (consulta del 1 de febrero de 2015). Véase también Emilio Godoy, "China-Mexico Ties Grow Thin”, Inter Press Service, IPS, 22 de enero de 2013, http:/ / www.ipsnews.net/2013/01/china-mexico-ties-grow-thin/ (consulta del 4 de febrero de 2014).

16 Véase Juan Carlos López, "Dragon Mart Cancun” (documento en línea), http://www.economia.unam.mx/deschimex/cechimex/chmxEx tras/documentos/actividades/Dragonmart/Juan_Carlos_Lopez_R.pdf (consulta del 30 de junio de 2018). 
cinco por ciento de una empresa privada china. ${ }^{17}$ De la misma manera, el siguiente embajador, Qiu Xiaoqi, afirmó a la prensa: "En este proyecto, en realidad, la mayoría son inversiones mexicanas; el gobierno chino no tiene nada que ver... Le tienen que preguntar esto a los inversionistas mexicanos, porque no conozco mucho este proyecto, simplemente no es de China". ${ }^{18}$ Probablemente su interés en ese momento estaba ya puesto en un negocio más jugoso y de largo plazo: el tren México-Querétaro.

\section{El caso del tren rápido Ciudad de México-Querétaro}

El segundo proyecto de China en el país, en el sexenio estudiado, fue el del tren rápido Ciudad de México-Querétaro, mucho más importante tanto por su tamaño como por la manera simbólica en que China lo veía, en términos del impacto que podría tener en la región. La obra se definía como un proyecto de vía de comunicación tipo ferroviario para pasajeros, que conectaría la Ciudad de México, partiendo de la estación terminal Buenavista hacia la Ciudad de Santiago de Querétaro, ciudad esta última que requeriría la construcción de una nueva estación. El proyecto mostraba que el tren pasaría por el territorio de cuatro entidades: el (entonces) Distrito Federal, Estado de México, Hidalgo y Querétaro, y en cada una, por distintos municipios y delegaciones. Estaba programado para realizarse en un periodo de 52 meses, desde la preparación del sitio hasta propiamente la construcción; se consideraba además una vida útil de al menos 30 años durante la operación del proyecto, según el estu-

17 "Calderón corrió a China de México", SIPSE.com, 3 de junio de 2013, https://sipse.com/mexico/calderon-corrio-a-china-de-mexico-346 07.html (consulta del 6 de septiembre de 2014).

18 Jesús Vázquez, "Se deslinda China del Dragon Mart", El Economista, 25 de noviembre de 2013, https://www.eleconomista.com.mx/estados/ Se-deslinda-China-del-Dragon-Mart-20131125-0055.html (consulta del 14 de diciembre de 2014). 
dio elaborado por el Dr. Guillermo Julio Román Moguel, bajo el esquema de licitación de obra pública federal. El monto de la inversión como anteproyecto se estimó en 40767.54 millones de pesos mexicanos. ${ }^{19}$

Desde el inicio del sexenio, bajo el lema de "relanzar las relaciones con China", los proyectos de infraestructura fueron parte de la "nueva" relación, que había decaído mucho por el recibimiento del presidente anterior, Felipe Calderón, al Dalai Lama. Estas obras eran y son parte fundamental de la política estratégica de China de invertir en infraestructura en casi todo el mundo, y particularmente en comunicaciones, de modo que, en general, desde China se veía como un proyecto emblemático para el resto de América Latina.

En una entrevista escrita concedida a la prensa china, con motivo de su asistencia al Foro Boao del 6 al 8 de abril de 2014, el presidente mexicano enfatizó que la cooperación bilateral tenía enormes oportunidades en comercio e infraestructura. Asimismo, sugirió la cooperación más estrecha a través de mecanismos bilaterales como el Grupo de Alto Nivel México China establecido en 2004. "La expansión de los Institutos Confucio en México, abriendo sucursales en más ciudades sería el camino perfecto para elevar una mayor conciencia de la cultura china entre los mexicanos y promover el estudio del mandarín". También dijo que México debería aprender de la experiencia de China en energía y desarrollo de infraestructura dado que China posee la red más grande de trenes bala. "China es, sin duda, un excelente modelo en ese tema... tenemos mucho que aprender de su exitosa historia en infraestructura de trenes. [...] El Ministerio de Comunicaciones y Transporte de México está actualmente trabajando en un plan de cinco años (2013-2018) para

${ }^{19}$ Véase Secretaría de Comunicaciones y Transportes, "Manifestación de impacto ambiental modalidad regional para el proyecto denominado tren de pasajeros México-Querétaro", (s.p.i), http://www.sct.gob.mx/fileadmin/DireccionesGrales/DGTFM/Proyectos_Pasajeros/Mex-Qro/ MIA-R_TMxQ_15012014.pdf (consulta del 6 de julio de 2018). 
construir una serie de ferrocarriles". ${ }^{20}$ Cinco meses después, el 4 de septiembre de 2013, el entonces secretario de Hacienda, Luis Videgaray, ${ }^{21}$ y el titular de la Comisión Nacional de Desarrollo y Reforma de China, Xu Shaoshi, firmaron en San Petersburgo un "Memorándum de entendimiento" sobre la promoción de inversión entre ambos países, que obviamente incluía la construcción de infraestructura.

Las empresas chinas estaban muy entusiasmadas y habían invertido cerca de 2 millones de dólares en sus estudios. El 3 de noviembre 2014, la Secretaría de Comunicaciones y Transportes (SCT) informó que la licitación había sido ganada por China Railway y sus socios mexicanos: GIA, Prodemex, GHP y TEYA. El 6 de noviembre del mismo año se anunció la cancelación del trato, ante la publicación de una investigación periodística que revelaba conflictos de interés entre el presidente de México y empresas mexicanas asociadas con las empresas chinas: la China Railway Construction Corporation (CRCC) no construiría el tren. La convocatoria volvió a abrirse, pero ante las críticas y presiones se anunció su suspensión definitiva en enero de 2015, aduciendo carencias presupuestarias. Las compañías chinas involucradas y ganadoras de la primera licitación tuvieron una reacción muy negativa. Algunas fuentes afirman que allegados al ejecutivo mexicano recomendaron, en días previos a la presentación del proyecto a las compañías chinas, con cuáles compañías mexicanas debían asociarse. Esto llevó al escándalo de corrupción desatado por la participación de compañías vinculadas con el presidente de la Republica y su funcionario más cercano, Luis Videgaray. ${ }^{22}$

20 "Interview: Mexico sees China as «a strategic partner»: President", China Daily, 4 de abril de 2013, http:/ / www.chinadaily.com.cn/business/20 13-04/04/content_16376594.htm (consulta del 15 de noviembre de 2015).

${ }^{21}$ Luis Videgaray fue secretario de Hacienda del $1^{\circ}$ de diciembre de 2012 al 7 de septiembre de 2016 y posteriormente secretario de Relaciones Exteriores, del 4 de junio de 2017 al 30 de noviembre de 2018.

${ }_{22}^{2}$ Para ver un recuento más puntual de este caso, véase Ernesto Cordero Arroyo, "Proposición con un punto de acuerdo que exhorta al ejecutivo 
Ciertamente, el Secretario de Hacienda, Luis Videgaray, encabezó un "Grupo de Trabajo de Alto Nivel" con el gobierno de China cuyo proyecto principal fue realizar el Tren de Alta Velocidad México-Querétaro, once meses antes del lanzamiento de la licitación, y sostuvo varias reuniones con el citado grupo. Debido a esto, el chino fue el único consorcio que presentó una propuesta; las demás empresas interesadas (incluyendo firmas internacionales como CAF, Mitsubishi, Bombardier, Alstom y Siemens) pidieron una ampliación del plazo para entregar su oferta y, al no terminarla, enviaron cartas de disculpas.

Los informes periodísticos hicieron eco de la denuncia de corrupción que supuestamente fue investigada por Virgilio Andrade, quien fuera nombrado titular de la Secretaría de la Función Pública ( $\mathrm{SFP}$ ) por el propio presidente Peña Nieto, para que lo investigara a él mismo y a los miembros más allegados de su equipo.

Un elemento crucial en este asunto fue el excelente y bien documentado reportaje presentado por la periodista Carmen Aristegui el 9 de noviembre de 2014, en el cual demostró que el presidente Peña Nieto vivía en una casa de Juan Armando Hinojosa Cantú, propietario de Constructora Teya. Asimismo, su seguimiento posterior del caso ha sido ejemplo de periodismo de investigación. ${ }^{23}$ El 11 de diciem-

federal a explicar las razones por las que dejó sin efecto la construcción del tren de alta velocidad México-Querétaro", Grupo Parlamentario del PAN, Senado de la República, 15 de febrero de 2018, http://sil.goberna cion.gob.mx/Archivos/Documentos/2018/02/asun_3667482_20180220 _1518715689.pdf (consulta del 13 de octubre de 2018).

23 Sebastián Barragán, "Videgaray dirigió a China en propuesta del Tren México-Querétaro, revela documento", Aristegui Noticias, 6 de febrero de 2018, https://aristeguinoticias.com/0602/mexico/videgaraydirigio-a-china-en-propuesta-del-tren-mexico-queretaro-revela-documen to/ (consulta del 7 de febrero de 2018); también véase "Aviso de intención de someter a la reclamación a arbitraje y del comienzo del periodo para celebrar consultas amistosas o negociaciones, conforme al artículo 12 del Acuerdo entre el gobierno de los Estados Unidos Mexicanos y el gobierno de la República Popular China para la Promoción y Protección Recí- 
bre del mismo año, The Wall Street Journal dio a conocer que Luis Videgaray compró una casa a Hinojosa en Malinalco, Estado de México, con valor de 7.5 millones de pesos. ${ }^{24}$ En noviembre de 2017, Aristegui Noticias informó que, en este proceso, China exigía un pago de 600 millones de dólares, lo que corresponde a 11000 millones de pesos al tipo de cambio vigente en octubre de 2015 .

De esa manera terminó el idilio inicial entre ambos países y el "relanzamiento" de las relaciones entre México y China. Las posturas autoritarias del ejecutivo mexicano, acostumbrado a la impunidad, hicieron creer al gobierno chino que podrían asegurar negocios desde arriba, como en África, pero en este caso, el excelente trabajo periodístico de Carmen Aristegui y su equipo filtró la verdad en el híbrido sistema político mexicano, aun si como represalia pagaron por ello con su propio espacio informativo, dado que la empresa de medios la demandó en un juicio que duró casi cuatro años, con montos elevados de por medio, que finalmente ganó la periodista. En medio de este asunto, la desaparición, aún no resuelta, de 43 estudiantes en Ayotzinapa en septiembre de 2014, rompió para el resto del sexenio la credibilidad del gobierno para la mayoría de la población.

\section{DE LAS RELACIONES DIPLOMÁTICAS}

En el ámbito de la diplomacia, las relaciones en un primer momento fueron muy cercanas. En el estilo chino, fueron elevadas al nivel de "asociación estratégica integral" (si bien no es claro lo que esto significa) y, particularmente, se establecie-

proca de las Inversiones" (s/1), 9 de octubre de 2015, https://issuu.com/ aristeguinoticias/docs/aviso_de_intenci_n_china_railway (consulta del 3 de octubre de 2018).

${ }^{24}$ Juan Montes, "Mexico Finance Minister Bought House from Government Contractor", The Wall Street Journal, 11 de diciembre de 2014, https:/ / www.wsj.com/articles/new-ties-emerge-between-mexico-govern ment-and-builder-1418344492 (consulta del 12 de diciembre de 2014). 
ron muchos mecanismos de diálogo en casi cada nivel de la relación. Los presidentes Xi Jinping y Enrique Peña Nieto se reunieron siete veces entre visitas mutuas y encuentros en foros multilaterales.

México, junto con Perú, fue muy protagónico en la aspiración de China de tener un mecanismo de diálogo regional. De esa manera, se logró establecer el Foro de la Comunidad de Estados Latinoamericanos y Caribeños (Celac) y China. Su primera reunión anual se celebró en Beijing, el 8 y 9 de enero de 2015, con varios subforos para áreas específicas: Foro de Ministros de Agricultura China, Foro de Innovación Científico-Tecnológica, Cumbre Empresarial China-América Latina y el Caribe, Foro de Intercambio de Think Tanks, Foro de Políticos Jóvenes, Foro de Cooperación en Infraestructura, Foro de Partidos Políticos, Foro de Amistad entre Sociedades Civiles China-América Latina y el Caribe. Además, en la más reciente reunión en 2018, China ofreció a la región los beneficios de inversión de su proyecto "Un cinturón una ruta”, sobre el cual se firmó un acuerdo. Independientemente de la eficacia de la Celac, éste ha sido un espacio importante en el cual una región tan fragmentada se ha reunido sin la presencia de Estados Unidos y Canadá, lo cual en la perspectiva racional de China es un mecanismo importante para lograr acuerdos con la región, aprovechando las políticas centradas en sí mismo del gobierno estadounidense.

Por su parte el gobierno de Estados Unidos ha observado con atención y desconfianza la creciente presencia de China en América Latina. El $1^{\circ}$ de febrero de 2018, el secretario de Estado de Estados Unidos en ese momento, Rex Tillerson, comenzó una gira de siete días por varios países de América Latina y el Caribe. Los países que visitó Tillerson fueron México, Argentina, Perú, Colombia y Jamaica. Las visitas se centraron en "Promover un hemisferio seguro y próspero con seguridad enérgica y democrática” ${ }^{25}$ El punto de partida de

${ }^{25}$ Rex Tillerson, "U.S. Engagement in the Western Hemisphere" (discurso), Austin, University of Texas, , 1 de febrero de 2018, https://www. 
la gira Tillerson fue su visita a la Universidad de Austin en Texas, su alma mater, donde describió las prioridades políticas para la administración actual en relación con los asuntos del llamado hemisferio occidental.

Durante una sesión de preguntas y respuestas, después de su discurso en Austin, Tillerson elogió la Doctrina Monroe de 1823 (América para los estadounidenses) como "claramente [...] un éxito". La doctrina y su consiguiente corolario, que data de 1904, a cargo del presidente Theodore Roosevelt, afirman la autoridad de Estados Unidos en el hemisferio occidental, por encima de la intromisión de las potencias europeas. Tillerson dijo en referencia al documento: "Creo que hoy es tan relevante como lo fue el día en que fue escrita”. Sus comentarios eran un repudio directo al enfoque de la administración de Barack Obama en la región, cuando en 2013, el predecesor de Tillerson, John Kerry, declaró que "la era de la Doctrina Monroe había terminado".

Tillerson advirtió sobre las ambiciones comerciales "imperiales" de China y promovió a Estados Unidos como el socio comercial preferido de la región. También pintó a China como una espada para Estados Unidos: "Hoy China está estableciendo sus bases en América Latina. Está utilizando el arte de gobernar en lo económico para llevar a la región a su órbita. La pregunta es: ¿a qué precio?”. Y agregó: “América Latina no necesita nuevos poderes imperiales que sólo buscan beneficiar a su propio pueblo [...] El modelo de desarrollo liderado por el Estado chino recuerda al pasado [...] No tiene que ser el futuro de este hemisferio". También expresó sus deseos de que Washington siguiera siendo el "socio más firme, fuerte y duradero de la región”. ${ }^{26}$ El siguiente se-

state.gov/secretary/20172018tillerson/remarks/2018/02/277840.htm (consulta del 14 de junio de 2018).

${ }^{26}$ Robbie Gramer y Keith Johnson, "Tillerson Praises Monroe Doctrine, Warns Latin America of 'Imperial' Chinese Ambitions. The Secretary of State Kicks off his Multicountry Tour trying to get the Region to Rally Behind Trump", Foreign Policy, 2 de febrero de 2018, citado en Romer Cornejo, "El proyecto Una Franja, Una Ruta de China y América Latina, 
cretario de Estado, Mike Pompeo, se ha reunido varias veces con las autoridades del nuevo gobierno de México, pero no desconocemos el contenido de las reuniones.

Es importante recalcar que, como apunta Dussel Peters, el recientemente acordado Acuerdo entre Canadá, Estados Unidos y México (USMCA, por sus siglas en inglés), que ya firmaron los presidentes en Buenos Aires en el marco del G20, pero que no ha sido ratificado por los congresos de los tres países, en

el artículo 32.10, exige que cualquier país del usmca que busque iniciar negociaciones de libre comercio con otro país de «no-mercado»-definido según cada uno de los miembroséste deberá informar con antelación a los demás miembros del usmca sobre sus objetivos y el potencial impacto del nuevo acuerdo, incluso si las negociaciones fueran confidenciales, con el objeto de que el USMCA se convirtiera en un acuerdo bilateral y se cancelara la participación del miembro del USMcA que busque el nuevo TLC. El artículo 32 [...] tiene claramente una dedicatoria a la República Popular China, es decir, que si uno de los miembros inicia negociaciones comerciales con China, este país muy probablemente sea dado de baja del [Acuerdo]. En la práctica, el usmca se convierte en un «bloque regional anti-China», el primero de estas características a nivel internacional y coherente con la visión geoestratégica de Trump. ${ }^{27}$

En cuanto a las relaciones culturales, China ha financiado un buen número de becas para mexicanos, además de invitar a personajes de todos los ámbitos de su interés. La mayo-

entre las posibilidades y la realidad", en Savino Vaca Narvaja y Zou Zhan (eds.), China y América Latina en la Geopolítica de la Nueva Ruta de la Seda, Buenos Aires, Universidad Nacional de Lanús, 2018, pp. 59-80.

27 Tomado casi a la letra de Enrique Dussel Peters, "usmca, ¿primer tratado internacional antichino?", Reforma, 30 de noviembre de 2018, http:/ / www.dusselpeters.com/reforma2018-07.pdf (consulta del 3 de diciembre de 2018). 
ría de ellos regresan deslumbrados por el desarrollo del país y por la generosidad de sus anfitriones. En este sentido, vale la pena leer el texto de Roger Bartra, escrito después de uno de sus viajes, donde relata sus conversaciones con algunos de los sociólogos y otros intelectuales con los que se entrevistó, todos ellos miembros probados del Partido Comunista, precisamente en 2014, año en que el gobierno había limitado bastante las discusiones académicas y sobre derechos humanos. ${ }^{28}$

De igual modo, innumerables actividades culturales oficiales de China se han llevado a cabo en universidades, museos, en el recinto del Senado y en otros foros en México. En este ámbito, vale la pena mencionar la exposición sobre Tíbet que se inauguró en el Museo Nacional de las Culturas del Mundo, el 15 de abril de 2018, "Tashi Delek, Semana Cultural de Xizang, China 2018”. Contó con la presencia del embajador chino en México, Qiu Xiaoqi, la secretaria técnica del Instituto Nacional de Antropología e Historia (INAH) de México, Aída Castilleja González, la directora del museo, Gloria Artís Mercadet, otros diplomáticos acreditados en México, y otros invitados. ${ }^{29} \mathrm{El}$ evento tuvo muy poca difusión probablemente con el propósito de no exaltar a los defensores de los derechos humanos de Tíbet. Cabe mencionar que el embajador de China en México, Qiu Xiaoqi, ha llevado a cabo una excelente labor en medios de difusión; son muy frecuentes sus artículos en la prensa, explicando y defendiendo, como corresponde, la política de su país. Es frecuente encontrarlo inaugurando ciclos de conferencias en las más importantes universidades, así como en la reunión anual

${ }^{28}$ Roger Bartra, De la Revolución a la Armonía. Diario de un viaje de estudios a China, México, unAm (Cuadernos de la Coordinación de Humanidades 2), 2016, https:/ / coordenadas2050.files.wordpress.com/2017/02/ 02_roger-bartra.pdf

${ }^{29}$ Véase "Embajador chino asiste a inauguración de la Semana Cultural de Xizang”, Embajada de la República Popular China en México (sitio de internet), 18 de abril de 2018, http:/ / mx.china-embassy.org/esp/sgjs / t1551961.htm (consulta del 30 de octubre de 2018). 
más reciente de la Asociación Mexicana de Estudios Internacionales, donde no era frecuente la presencia de embajadores de otros países. Sin duda, la generosidad de China con la comunidad académica ha rendido buenos frutos para formar opiniones favorables sobre ese país y evitar al máximo los temas considerados sensibles por su gobierno. Sus viajes de estudio han servido para habilitar a muchos universitarios como conocedores de China.

\section{Consideraciones FinALES}

Las relaciones de México con China en el sexenio 2012-2018 retrataron el comportamiento de los gobernantes políticos mexicanos: iniciaron con gran ímpetu, con relaciones personales muy cordiales entre los líderes, encuentros frecuentes de alto nivel e importantes proyectos económicos y, a mediados del sexenio, sucumbieron producto de la corrupción y la impunidad mexicanas, con un gobierno debilitado por la desaparición de 43 estudiantes normalistas y su incapacidad de resolver ése y muchos otros casos que comprometieron seriamente la gobernabilidad.

En el plano económico, el intercambio comercial siguió aumentando sin cambios significativos en el déficit de México, que en 2015 hizo varias investigaciones antidumping.

Paralelamente, las instituciones gubernamentales como la Secretaría de Relaciones Exteriores y la Secretaría de Economía cumplieron muy bien su trabajo, llevando a cabo innumerables reuniones puntuales de trabajo con China, y logrando firmar una serie de acuerdos en muchas ramas de la relación los cuales quedan como instrumentos institucionales en caso de que quisieran aprovecharse en el futuro.

Por su parte, con la ayuda de México, China obtuvo la constitución del Foro China-Celac, que si bien ha avanzado con lentitud, es posible que el retraimiento de Estados Unidos de la región le permita recuperar dinamismo en el futuro. Asimismo, continuó con una muy exitosa política cul- 
tural, logrando influir en los medios universitarios y políticos a favor de sus intereses y en el mejoramiento de su imagen.

\section{Bibliografía y DOCUMENTOS}

"Aviso de intención de someter a la reclamación a arbitraje y del comienzo del periodo para celebrar consultas amistosas o negociaciones, conforme al artículo 12 del Acuerdo entre el gobierno de los Estados Unidos Mexicanos y el gobierno de la República Popular China para la Promoción y Protección Recíproca de las Inversiones" (s/l), 9 de octubre de 2015, https:/ / issuu.com/aristeguinoticias/docs/aviso_de_intenci_n_chi na_railway (consulta del 3 de octubre de 2018).

Chang, Bao, "Dragon Mart to land in Cancun", China Daily, 7 de junio de 2013, http://www.chinadaily.com.cn/china/2013xivi sit/2013-06/07/content_16580627.htm (consulta del 3 de julio de 2013).

BARRAGán, Sebastián, "Videgaray dirigió a China en propuesta del Tren México-Querétaro, revela documento”, Aristegui Noticias, 6 de febrero de 2018, https: / / aristeguinoticias.com/0602/ mexico/videgaray-dirigio-a-china-en-propuesta-del-tren-mexi co-queretaro-revela-documento/ (consulta del 7 de febrero de 2018).

BARTRA, Roger, "De la Revolución a la Armonía. Diario de un viaje de estudios a China", Cuadernos de la Coordinación de Humanidades núm. 2. UNAM, México, 2016, https://coordenadas20 50.files.wordpress.com/2017/02/02_roger-bartra.pdf

“Calderón corrió a China de México", 3 de junio de 2013, https:/ / sipse.com/mexico/calderon-corrio-a-china-de-mexico-34607. html (consulta del 6 de septiembre de 2014).

Cordero Arroyo, Ernesto, "Proposición con punto de acuerdo que exhorta al ejecutivo federal a explicar las razones por las que dejó sin efecto la construcción del tren de alta velocidad México-Querétaro", Grupo Parlamentario del Pan, Senado de la República, 15 de febrero de 2018, http://sil.gobernacion.gob.mx/Archivos/Documentos/2018/02/asun_366748 
2_20180220_1518715689.pdf (consulta del 13 de octubre de 2018).

Connejo, Romer, "El proyecto «Una Franja, Una Ruta» de China y América Latina, entre las posibilidades y la realidad”, en Savino Vaca Narvaja y Zou Zhan (eds.), China y América Latina en la Geopolítica de la Nueva Ruta de la Seda, Universidad Nacional de Lanús, Buenos Aires, 2018, pp. 59-80.

Chávez, Víctor, "Cancelación de Dragon Mart, en respuesta a demanda ciudadana: PRD”, El Financiero, 26 de enero de 2015, http:/ / www.elfinanciero.com.mx/economia/cancelacion-dedragon-mart-en-respuesta-a-demanda-ciudadana-prd (consulta del 27 de enero de 2015).

"China-Mexico ties face unprecedented opportunity: Xi", China Daily, 6 de junio de 2013, http://www.chinadaily.com.cn/chi na/2013xivisit/2013-06/06/content_16577448.htm (consulta del 3 de julio de 2013).

"Dragon Mart Cancún: la destrucción del monstruo chino llegó a México", 21 de noviembre de 2012, https:/ / ecoosfera.com/20 12/11/dragon-mart-cancun-la-destruccion-del-monstruo-chi no-llego-a-mexico/ (consulta del 3 de diciembre de 2012).

Dussel Peters, Enrique, Coordinador, La relación México-China. Desempeño y propuestas para 2016-2018, México, unAM, Cámara de Comercio de México en China, Centro de Estudios ChinaMéxico, Unión de Universidades de América Latina y el Caribe, 2016, http:/ / www.dusselpeters.com/101.pdf (consulta del 7 de octubre de 2018).

Dussel Peters, Enrique, "usmca, ¿primer tratado internacional antichino?, Reforma, 30 de noviembre de 2018, Reforma, http:/ / www.dusselpeters.com/reforma2018-07.pdf (consulta del 3 de diciembre de 2018).

"Embajador chino asiste a inauguración de la Semana Cultural de Xizang”, Embajada de la República Popular China en México (sitio de internet), 18 de abril de 2018, http://mx.china-em bassy.org/esp/sgjs/t1551961.htm (consulta del 30 de octubre de 2018)

Gobierno de la República, Plan Nacional de Desarrollo 2013-2018. Diario Oficial de la Federación, 20 de mayo de 2013, http:// 
www.dof.gob.mx/nota_detalle.php?codigo $=5299465 \&$ fecha $=20 / 05 /$

Godoy, Emilio, “China-Mexico Ties Grow Thin”, Interpress Service, IPS News, 22 de enero de 2013, http://www.ipsnews.net/ 2013/01/china-mexico-ties-grow-thin/ (consulta del 4 de febrero de 2014).

Gramer, Robbie y Keith Johnson, "The secretary of state kicks off his multicountry tour trying to get the region to rally behind Trump”, Foreign Policy, 2 de febrero de 2018, https://foreignpolicy.com/2018/02/02/tillerson-praises-monroe-doctrinewarns-latin-america-off-imperial-chinese-ambitions-mexicosouth-america-nafta-diplomacy-trump-trade-venezuela-maduro/ (consulta del 30 de junio de 2018).

López, Juan Carlos, “Dragon Mart Cancun”, http:/ /www.econo mia.unam.mx/deschimex/cechimex/chmxExtras/documen tos/actividades/Dragonmart/Juan_Carlos_Lopez_R.pdf (consulta del 30 de junio de 2018).

McDonald, Ernestina, "Dragón Mart, la invasión china a Cancún", Noticieros Televisa, 6 de diciembre de 2012, http:/ / noticieros televisa.esmas.com/especiales/534081/dragon-mart-invasionchina-cancun/ (consulta del 15 de diciembre de 2012).

Montes, Juan, "Mexico Finance Minister Bought House From Government Contractor", The Wall Street Journal, 11 de diciembre de 2014, https://www.wsj.com/articles/new-ties-emerge-bet ween-mexico-government-and-builder-1418344492 (consulta del 12 de diciembre de 2014).

OJeDA, Marcela e Isabel Becerril, "Ven daño a relación MéxicoChina por Dragon Mart”, El Financiero, 27 de enero de 2015, http:/ / www.elfinanciero.com.mx/economia/ven-dano-a-rela cion-mexico-china-por-dragon-mart (consulta del $1^{\circ}$ de febrero de 2015).

Patiño, Dainzú, “¿Cómo reducir del déficit comercial de México con China?” Expansión, https://expansion.mx/economia/20 18/08/03/como-reducir-el-deficit-comercial-de-mexico-conchina (consulta del 12 de septiembre de 2018).

Peña Nieto, Enrique, "Interview: Mexico sees China as «a strategic partner»: President”, China Daily, 4 de abril de 2013, http:// 
www.chinadaily.com.cn/business/2013-04/04/content_ 16376594.htm (consulta del 15 de noviembre de 2015).

Presidencia de la República, $1^{\circ}$ Informe de Gobierno, septiembre de 2013.

Presidencia de la República, $2^{\circ}$ Informe de Gobierno, agosto de 2014.

Presidencia de la República, Plan Nacional de Desarrollo, Tercer informe de ejecución, marzo de 2016, https://www.gob.mx/ cms/uploads/attachment/file/73966/Informe_de_ejecuci _n.pdf

Presidencia de la República, $4^{\circ}$ Informe de Gobierno, agosto de 2016.

Presidencia de la República, $5^{\circ}$ Informe de Gobierno, agosto de 2017

Presidencia de la República, $6^{\circ}$ Informe de Gobierno, agosto de 2018

Restrepo, Iván, "Dragon Mart, negocio oscuro", La Jornada, 2 de febrero de 2015, https://www.jornada.com.mx/2015/02/02/ opinion/016a2pol (consulta del 3 de febrero de 2015).

Secretaría de Comunicaciones y Transportes, "Manifestación de impacto ambiental modalidad regional para el proyecto denominado tren de pasajeros México-Querétaro", (s.p.i), http:// www.sct.gob.mx/fileadmin/DireccionesGrales/DGTFM/Pro yectos_Pasajeros/Mex-Qro/MIA-R_TMxQ_15012014.pdf (consulta del 6 de julio de 2018).

Saucedo López, José David, "Posibles efectos de la implantación de la Alta Velocidad Ferroviaria en México: el caso del Tren Rápido de Pasajeros México - Querétaro”, México, UAm Azcapotzalco, tesis de Maestría, s/f, https://core.ac.uk/download/ pdf/128743861.pdf (consulta del 20 de octubre de 2018).

Tillerson, Rex, "U.S. Engagement in the Western Hemisphere" (discurso), Austin, University of Texas, 1 de febrero de 2018, https://www.state.gov/secretary/20172018tillerson/re marks/2018/02/277840.htm (consulta del 14 de junio de 2018).

VÁzQuez, Jesús, "Se deslinda China del Dragon Mart", El Economista, 25 de noviembre de 2013, https://www.eleconomista.com. 
$\mathrm{mx} /$ estados/Se-deslinda-China-del-Dragon-Mart-20131125 0055.html (consulta del 14 de diciembre de 2014).

YUANYUAN, Hu, "Plans afoot to open second «Dragon Mart» commercial center in Mexico", China Daily, 23 de febrero de 2012, http://global.chinadaily.com.cn/a/201202/23/WS5a2f5 468a3108bc8c6723337.html (consulta del 14 de julio de 2018). 